\title{
The association between health insurance status and utilization of health services in rural Northern Ghana: evidence from the introduction of the National Health Insurance Scheme
}

Philip Ayizem Dalinjong ${ }^{1 *}$, Paul Welaga1, James Akazili, ${ }^{1,3}$, Anthony Kwarteng², Martin Bangha ${ }^{3}$, Abraham Oduro $^{1}$, Osman Sankoh ${ }^{3}$ and Jane Goudge ${ }^{4}$

\begin{abstract}
Background: Many households in low- and middle-income countries face financial hardships due to payments for health care, while others are pushed into poverty. Risk pooling and prepayment mechanisms help to lessen the impact of the costs of care as well as assisting to achieve universal health coverage (UHC). Ghana implemented the National Health Insurance Scheme (NHIS) for the promotion of access to health services for all Ghanaians. In this paper, we examined the association between health insurance status and utilization of outpatient and inpatient health services in rural poor communities.
\end{abstract}

Methods: The study was a cross-sectional household survey conducted in the Kassena-Nankana districts of Northern Ghana. We conducted interviews in 11,175 households and collected data on 55,992 household members. Multiple logistic regression models were used to identify factors associated with the utilization of outpatient and inpatient health services. The dependent variables were the utilization of outpatient and inpatient health services. We adjusted for several potential socio-demographic factors associated with utilization and health insurance status.

Results: Significantly, the insured had 2.51 (95\% Cl 2.3-2.8) and 2.78 (95\% Cl 2.2-3.6) increased odds of utilizing outpatient and inpatient health services respectively. Respondents with a history of recent illness or injury [32.4 (95\% Cl 29.4-35.8) and $5.72(95 \% \mathrm{Cl} 4.6-7.1)]$ and poor or very poor self-reported health status [2.08 (95\% Cl 1.7-2.5) and $2.52(95 \% \mathrm{Cl} 1.9-3.4)]$ and those on chronic medication $[2.79(95 \% \mathrm{Cl} 2.2-3.5)$ and $3.48(95 \% \mathrm{Cl} 2.5-4.8)]$ also had increased odds of utilizing both outpatient and inpatient health services respectively. Among the insured, the poorest use the Community-based Health Planning and Services (CHPS) compounds, while the least poor use private clinics and public hospitals for outpatient health services. The uninsured predominately use pharmacies or licensed chemical shops (LCSs). For inpatient health services, the insured largely use public hospitals, with the uninsured using private clinics or public health centres.

Conclusion: The findings suggest that being insured with the NHIS is associated with increased utilization of outpatient and inpatient health services in the study area. Overall, the NHIS can be an effective tool for achieving UHC and hence pragmatic efforts should be made to sustain it.

Keywords: National Health Insurance, Social health insurance, Health service, Utilization, Outpatients, Inpatients, Insured, Uninsured, Ghana

\footnotetext{
* Correspondence: padalinjong@yahoo.com

${ }^{1}$ Navrongo Health Research Centre, Ghana Health Service, Navrongo, Ghana

Full list of author information is available at the end of the article
} 


\section{Background}

Access to quality health services is problematic for most people in the world. The World Health Organization (WHO) reports that about 400 million people globally, do not have access to basic quality health services and that $6 \%$ of people living in low- and middle-income countries experience extreme poverty as a result of payments for health services [1]. Universal health coverage (UHC), defined as access to basic quality health services without financial hardship, can assist in alleviating poverty. The concept is firmly rooted in the $1948 \mathrm{WHO}$ Constitution; which declared health as a fundamental human right [2]. Given the importance of UHC, it has been made one of the targets for the sustainable development goals (goal 3) initiated by the United Nations in September, 2015. The target calls on all countries to endeavor to achieve UHC by year 2030. As a result, many countries including low and middle ones are pursuing various health sector reforms to pave the way for attaining UHC. These are done through the introduction of risk pooling and prepayment programs. In Africa for instance, Ghana, Rwanda, Nigeria, and Kenya have introduced health insurance schemes to allow their populations to have access to basic quality health services when needed.

\section{The National Health Insurance Scheme in Ghana}

Ghana established a National Health Insurance Scheme (NHIS) through an Act of Parliament (Act 650) and it began full operations in 2005 [3]. The core objective of the NHIS is the provision of quality health services for all Ghanaians without any out-of pocket (OOP) payments at the point of care $[4,5]$. The NHIS is functional in all metropolitan, municipal, and districts in Ghana. It is mandatory for all Ghanaians to register with the NHIS, to be able to have access to health services when needed, although this is hardly enforced. Financing sources of the NHIS include contributions from informal sector workers, the National Health Insurance levy, (a $2.5 \%$ top up on the value added tax), 2.5\% deduction from the contributions of formal sector workers to the Social Security and National Insurance Trust (SSNIT), and returns on investment from the National Health Insurance Fund [4]. The minimum and maximum contributions from the informal sector to the NHIS range between GH\$7.20 and 47.70 (\$8-\$53) [4]. However, vulnerable groups such as pregnant women, pensioners, children below the age of 18 years, and adults above the age of 70 are all exempted from premium payment [4].

Despite such policies, studies on health insurance programs in Africa have demonstrated that the poor are not covered [6]. In Ghana for instance, evidence showed that the NHIS is not pro-poor [7-11], despite the fact that the NHIS was established as part of a poverty reduction strategy and subsequently provided exemptions for the poor [12].

Poverty has been shown to be a profound barrier to registration with the NHIS in the three regions of Northern Ghana (Upper West region, Upper East region, and Northern region) which are considered as rural and poor, compared to the rest of the country [10, 13-15]. There is limited knowledge on the effect of the NHIS on utilization of health services from rural poor communities, particularly whether health insurance enables higher utilization. Thus, this study examined the utilization of both outpatient and inpatient health services in a rural poor setting after a decade of the implementation of the NHIS. The findings will assist to understand the progress made so far in the move towards UHC, particularly the extent to which the poor have access to health services. The results are also essential for policy makers in their formulation of interventions for the poor and vulnerable.

\section{Methods \\ Study area and design}

The study was a cross-sectional household survey conducted in the Kassena-Nankana districts (East and West) of the Upper East region of Ghana. The study area has a continuous population registration system known as the Navrongo Health and Demographic Surveillance System (NHDSS). The NHDSS monitors the demographic events of the entire population of about 160,000 in 30,000 households. Data on demographic events such as births, deaths, migrations, marriages, and pregnancies are collected and updated every 3 to 4 months. Agriculture and related activities are the mainstay of the economy of the districts, with about $78.9 \%$ of the population engaged in the sector for the year 2008 [16]. Due to the dependence on agriculture and declining agricultural yields, poverty is endemic in the area [17]. Health facilities in the study area include a district hospital, 6 health centres, 27 Community-based Health Planning and Services (CHPS) compounds, 2 mission-based health centres, and 3 private clinics. The CHPS compounds are community-based health centres that are meant to provide basic primary health services for individuals in rural and remote communities. At the time of the study, the only hospital in the two districts was located in the Kassena-Nankana East district, with a 140bed capacity. The main health centre (currently being upgraded to a district hospital) in the Kassena-Nankana West district had a 40-bed capacity. Doctor to patient ratio for the two districts was 1:31,927 in 2007 [16]. Malaria has consistently been the number one cause of outpatients' attendance. Other top health problems include respiratory tract infections and skin and diarrheal diseases [16]. The study was conducted from July to December 2012, using a structured questionnaire. 


\section{Sampling}

In all, 12,000 households were sampled from the total number of households in the NHDSS database using a simple random sampling technique. The sampling was done using STATA 12.0 [18]. Each sampled household was visited by a fieldworker who interviewed the household head or any adult member of the household who had information on the health insurance status of the household members and their health-seeking behavior and payments for health services. We collected data on 55,992 household members from 11,175 households in the study area.

\section{Study variables}

The dependent variables were the utilization of outpatient or inpatient health services. Outpatient utilization of service was defined as the use of any health service or purchase of drugs by an individual in the household in the last 1 month, ending on the day of interview for the study. The hospitalization of any member of the household in the last 1 year was considered as inpatient utilization, which included the day the interview was conducted. The independent variables included the socio-economic and demographic characteristics of the respondents such as wealth status, age, gender, educational status, religious affiliation, ethnicity, health insurance, geographical location, and household size. Household members with valid health insurance cards at the time of the interview were considered as being insured with the NHIS. Socio-economic status was computed from large and small household items using principal component analysis and accordingly categorized as poorest, poorer, poor, less poor, and the least poor.

\section{Data analysis and management}

Data was entered in FoxPro 6.0 and analysed in STATA 12.0 [18]. Multiple logistic regression models were used to identify factors associated with the utilization of outpatient and inpatient health services. The inclusion of variables in the regression model was based on their level of significance $(p<0.05)$ in the bivariate analysis as well as on scientific plausibility. Reference categories were defined as those usually associated with the least utilization of health services in the literature.

\section{Results}

\section{Socio-demographic characteristics of respondents}

The response rate for the study was $93 \%$. About $63 \%$ of household members were insured and 37\% uninsured. Respondents who were most likely to be insured were those who were sick in the last month, as well as those with poor or very poor self-reported health status (Table 1). Women were also more likely to be insured, as were young children (0-4), those living in urban areas, the least poor, and those with tertiary education.

\section{Utilization of outpatient and inpatient health services}

The insured made more outpatient as well as inpatient visits compared to the uninsured (Table 2). The same trend was observed for the utilization of inpatient health services. Equally, respondents with a history of recent illness/injury, those with poor or very poor selfassessed health status as well as those on chronic medication had more visits for both outpatient and inpatient health services. However, no difference was seen in the use of health services for the various socio-economic groupings.

\section{Factors associated with utilization of outpatient and inpatient health services}

Significantly, the insured had 2.51 (95\% CI 2.3-2.8) and 2.78 (95\% CI 2.2-3.6) increased odds of utilizing both outpatient and inpatient health services respectively, when compared to the uninsured (Tables 3 and 4). Further, respondents with a history of recent illness or injury and poor or very poor self-assessed health status and those on chronic medication had increased odds of utilizing both outpatient and inpatient health services. But poverty was not seen to be a significant factor in the utilization of outpatient health services (Table 3), except for inpatient health services. For instance, the least poor had 2.10 (95\% CI 1.5-2.9) increased odds of using inpatient health services compared to the poorest.

\section{Health insurance status and types of health providers visited}

We examined the different types of health providers utilized by respondents for both outpatient and inpatient health services (Table 5). Among the insured, there was some variation as to which facilities were utilized for outpatient health services, with the poorest using CHPS compounds. The least poor used private clinics and public hospitals instead, for their outpatient health needs. But the uninsured predominately use pharmacies or licensed chemical shops (LCSs). These facilities are solely responsible for the sale of drugs to clients. For inpatient health services, the insured largely use public hospitals, although utilization by the least poor is more than double that of the poorest. Within the uninsured, utilization of inpatient health service is much lower, with the poorest not using public hospitals at all, but rather private clinics or public health centres.

\section{Discussion}

The study examined the association between the insurance status of individuals and the utilization of outpatient and inpatient health services. It also examined 
Table 1 Background characteristics of study respondents by health insurance status

\begin{tabular}{|c|c|c|c|c|}
\hline \multirow[t]{2}{*}{ Variable } & \multirow[b]{2}{*}{ Categories } & \multirow{2}{*}{$\begin{array}{l}\text { Total } \\
\text { Number(\%) }\end{array}$} & \multirow{2}{*}{$\begin{array}{l}\text { Insured } \\
\text { Number(\%) }\end{array}$} & \multirow{2}{*}{$\begin{array}{l}\text { Uninsured } \\
\text { Number(\%) }\end{array}$} \\
\hline & & & & \\
\hline \multirow[t]{6}{*}{ Age } & $0-4$ & $5952(10.6)$ & $4410(74.1)$ & $1542(25.9)$ \\
\hline & $5-17$ & $17,765(31.7)$ & $11,299(63.6)$ & $6466(36.4)$ \\
\hline & $18-34$ & $13,957(24.9)$ & $8960(64.2)$ & 4997 (35.8) \\
\hline & $35-59$ & $12,352(22.1)$ & $7275(58.9)$ & $4077(41.1)$ \\
\hline & $60-69$ & $3467(6.2)$ & $1983(57.2)$ & $1484(42.8)$ \\
\hline & $70+$ (aged) & $2499(4.5)$ & $1422(56.9)$ & $1077(43.1)$ \\
\hline \multirow[t]{2}{*}{ Gender } & Male & $27,078(48.4)$ & $16,355(60.4)$ & $10,723(39.6)$ \\
\hline & Female & $28,914(51.6)$ & $18,996(65.7)$ & 9918 (34.3) \\
\hline \multirow[t]{5}{*}{ Educational level } & No formal education & $13,519(24.1)$ & $7354(54.4)$ & $6165(45.6)$ \\
\hline & Primary & $17,901(32.0)$ & $10,490(58.6)$ & $7411(41.4)$ \\
\hline & Secondary & $11,580(20.7)$ & $8118(70.1)$ & $3462(29.9)$ \\
\hline & Tertiary & $1659(3.0)$ & $1425(85.9)$ & $234(14.1)$ \\
\hline & Below school going age ${ }^{a}$ & $11,333(20.2)$ & 7967 (70.3) & $3366(29.7)$ \\
\hline \multirow[t]{3}{*}{ Religion } & Traditional \& other & $22,904(44.6)$ & $11,864(51.8)$ & $11,040(48.2)$ \\
\hline & Christianity & $27,737(49.6)$ & $19,471(70.2)$ & $8266(29.8)$ \\
\hline & Islam & $3260(5.8)$ & $2579(79.1)$ & $681(20.9)$ \\
\hline \multirow[t]{4}{*}{ Ethnicity } & Kasem & $28,099(50.2)$ & $20,091(71.5)$ & $8008(28.5)$ \\
\hline & Nankam & $23,393(41.8)$ & $11,977(51.2)$ & $11,416(48.8)$ \\
\hline & Buli & $1221(2.2)$ & $832(68.1)$ & $389(31.9)$ \\
\hline & Other & $3279(5.8)$ & $1024(86.2)$ & $164(13.8)$ \\
\hline \multirow[t]{2}{*}{ Location } & Rural & $48,562(86.7)$ & $28,992(59.7)$ & $19,570(40.3)$ \\
\hline & Urban & $7430(13.3)$ & $6360(85.6)$ & $1070(14.4)$ \\
\hline \multirow[t]{3}{*}{ Household size } & 1 member & $1267(2.3)$ & $780(61.6)$ & $487(38.4)$ \\
\hline & 2-4 members & $12,392(22.1)$ & $7980(64.4)$ & 4412 (35.6) \\
\hline & 5+ members & $42,333(75.6)$ & $26,585(62.8)$ & $15,748(37.2)$ \\
\hline \multirow[t]{5}{*}{ Socio-economic status } & Poorest & $15,211(27.1)$ & $7588(53.6)$ & $6568(46.4)$ \\
\hline & Poorer & $12,357(22.1)$ & $6182(54.7)$ & $5120(45.3)$ \\
\hline & Poor & $10,454(18.7)$ & $6063(58.0)$ & $4758(42.0)$ \\
\hline & Less poor & $10,821(19.3)$ & $7813(72.2)$ & $3008(27.8)$ \\
\hline & Least poor & 7149 (12.8) & $6270(87.7)$ & $879(12.3)$ \\
\hline \multirow[t]{2}{*}{ Recent illness or injury } & Yes & $2837(5.1)$ & $1770(62.4)$ & 1067 (37.6) \\
\hline & No & $53,155(94.9)$ & $33,594(63.2)$ & $19,561(36.8)$ \\
\hline \multirow[t]{4}{*}{ Self-assessed health status } & Poor or very poor & $1471(2.6)$ & $756(51.4)$ & $715(48.6)$ \\
\hline & Good or very good & $53,827(96.1)$ & $34,234(63.6)$ & $19,593(36.4)$ \\
\hline & Don't know & $694(1.3)$ & $376(54.2)$ & $318(45.8)$ \\
\hline & Overall total & $55,992(100)$ & $35,275(63 \%)$ & $20,717(37 \%)$ \\
\hline
\end{tabular}

${ }^{a}$ Representing infants who have not yet attained school going age in order to be in school

household members' utilization of various types of health providers. Being insured was significantly associated with higher odds of utilizing both outpatient and inpatient health services. The finding is consistent with other studies on utilization in other parts of Ghana and Africa $[19,20]$. A study in Accra, Ghana's capital city, showed that registered clients of the NHIS were significantly more likely to receive prescriptions, visit clinics, and use formal health facilities when in need [21]. Another study among households in the Mfantseman municipality (central region) in Ghana reported that insured members were more likely to utilize outpatient health services compared to the uninsured [22]. Studies in Rwanda have also shown an increase in the 
Table 2 Utilization rates for outpatient and inpatient health services

\begin{tabular}{|c|c|c|c|c|c|}
\hline \multirow[t]{2}{*}{ Variable } & \multirow[b]{2}{*}{ Categories } & \multicolumn{2}{|l|}{ Visits } & \multicolumn{2}{|c|}{ Utilization rate/per 1000} \\
\hline & & Outpatient & Inpatient & Outpatient & Inpatient \\
\hline \multirow[t]{2}{*}{ Health insurance status } & Insured & 3633 & 959 & 1.0 & 0.3 \\
\hline & Uninsured & 1364 & 101 & 0.7 & 0.1 \\
\hline \multirow[t]{6}{*}{ Age } & $0-4$ & 1033 & 194 & 1.7 & 0.3 \\
\hline & $5-17$ & 1104 & 180 & 0.6 & 0.1 \\
\hline & $18-34$ & 796 & 244 & 0.1 & 0.2 \\
\hline & $35-59$ & 1270 & 302 & 1.0 & 0.2 \\
\hline & $60-69$ & 457 & 78 & 1.3 & 0.2 \\
\hline & $70+$ (aged) & 337 & 62 & 1.4 & 0.3 \\
\hline \multirow[t]{2}{*}{ Gender } & Male & 2080 & 442 & 0.8 & 0.2 \\
\hline & Female & 2917 & 618 & 1.0 & 0.2 \\
\hline \multirow[t]{5}{*}{ Educational level } & No formal education & 1403 & 228 & 1.0 & 0.2 \\
\hline & Primary & 1186 & 199 & 0.7 & 0.1 \\
\hline & Secondary & 864 & 256 & 0.8 & 0.2 \\
\hline & Tertiary & 133 & 57 & 0.8 & 0.3 \\
\hline & Below school going age ${ }^{a}$ & 1411 & 320 & 1.3 & 0.3 \\
\hline \multirow[t]{3}{*}{ Religion } & Traditional \& other & 2070 & 295 & 0.9 & 0.1 \\
\hline & Christianity & 2478 & 659 & 0.9 & 0.2 \\
\hline & Islam & 241 & 62 & 0.7 & 0.2 \\
\hline \multirow[t]{4}{*}{ Ethnicity } & Kasem & 2344 & 581 & 0.8 & 0.2 \\
\hline & Nankam & 2128 & 349 & $.0-9$. & 0.2 \\
\hline & Buli & 219 & 35 & 1.8 & 0.3 \\
\hline & Other & 98 & 51 & 0.8 & 0.4 \\
\hline \multirow[t]{2}{*}{ Location } & Rural & 4244 & 747 & 0.9 & 0.2 \\
\hline & Urban & 753 & 313 & 1.0 & 0.4 \\
\hline \multirow[t]{3}{*}{ Household size } & 1 member & 159 & 43 & 1.3 & 0.3 \\
\hline & 2-4 members & 1256 & 305 & 1.0 & 0.3 \\
\hline & $5+$ members & 3582 & 712 & 0.9 & 0.2 \\
\hline \multirow[t]{5}{*}{ Socio-economic status } & Poorest & 1300 & 189 & 0.9 & 0.1 \\
\hline & Poorer & 972 & 145 & 0.8 & 0.1 \\
\hline & Poor & 867 & 149 & 0.8 & 0.1 \\
\hline & Less poor & 944 & 262 & 0.9 & 0.2 \\
\hline & Least poor & 704 & 271 & 0.9 & 0.4 \\
\hline \multirow[t]{2}{*}{ Recent illness or injury } & Yes & 2821 & 776 & 7.7 & $0-9$ \\
\hline & No & 2176 & 243 & 0.5 & 0.2 \\
\hline \multirow[t]{3}{*}{ Self-assessed health status } & Poor or very poor & 571 & 128 & 3.9 & 0.9 \\
\hline & Good or very good & 4371 & 886 & 0.8 & 0.2 \\
\hline & Don't know & 55 & 5 & 0.8 & 0.1 \\
\hline \multirow[t]{3}{*}{ Chronic medication } & Yes & 396 & 113 & 5.3 & 1.5 \\
\hline & No & 4591 & 903 & 0.8 & 0.2 \\
\hline & Don't Know & 10 & 3 & 0.8 & 0.3 \\
\hline
\end{tabular}

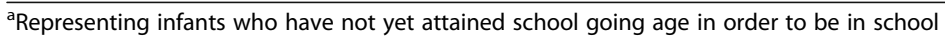


Table 3 Factors associated with utilization of outpatient health services

\begin{tabular}{|c|c|c|c|c|}
\hline \multirow[t]{2}{*}{ Variable } & \multirow[b]{2}{*}{ Categories } & \multicolumn{3}{|c|}{ Outpatient health services } \\
\hline & & $\%$ Utilized & Unadjusted OR (95\% Cl) & Adjusted OR $(95 \% \mathrm{Cl})$ \\
\hline \multirow[t]{2}{*}{ Health insurance status } & Insured & 7.9 & $2.06(1.9-2.3)$ & $2.51(2.3-2.8)^{b}$ \\
\hline & Uninsured & 3.9 & 1 & 1 \\
\hline \multirow[t]{6}{*}{ Age } & $0-4$ & 14.3 & $4.11(3.7-4.6)$ & $2.72(2.2-3.3)^{b}$ \\
\hline & $5-17$ & 4.5 & $1.17(1.0-1.3)$ & $1.19(1.0-1.4)$ \\
\hline & $18-34$ & 3.9 & 1 & 1 \\
\hline & $35-59$ & 6.9 & $1.85(1.7-2.1)$ & $1.55(1.4-1.8)^{b}$ \\
\hline & $60-69$ & 8.9 & $2.39(2.1-2.8)$ & $1.75(1.4-2.1)^{b}$ \\
\hline & $70+$ (aged) & 9.8 & $2.67(2.3-3.1)$ & $1.67(1.3-2.1)^{\mathrm{b}}$ \\
\hline \multirow[t]{2}{*}{ Gender } & Male & 5.6 & 1 & 1 \\
\hline & Female & 7.2 & $1.31(1.2-1.4)$ & $1.21(1.1-1.3)^{b}$ \\
\hline \multirow[t]{5}{*}{ Educational level } & No education & 7.2 & $1.29(1.0-1.6)$ & $1.01(0.8-1.3)$ \\
\hline & Primary & 4.4 & $0.77(0.6-0.9)$ & $0.93(0.7-1.2)$ \\
\hline & Secondary & 5.7 & $0.91(0.7-1.1)$ & $1.12(0.9-1.5)$ \\
\hline & Tertiary & 10.2 & 1 & 1 \\
\hline & Below school going age ${ }^{a}$ & 10.2 & $1.88(1.5-2.3)$ & $1.2(0.9-1.7)$ \\
\hline \multirow[t]{3}{*}{ Religion } & Traditional \& other & 6.4 & $1.10(0.94-1.29)$ & NS \\
\hline & Christianity & 6.5 & $1.10(0.9-1.3)$ & NS \\
\hline & Islam & 5.9 & 1 & NS \\
\hline \multirow[t]{4}{*}{ Ethnicity } & Kasem & 5.9 & 1 & 1 \\
\hline & Nankam & 6.5 & $1.09(1.0-1.2)$ & $1.33(1.2-1.5)^{b}$ \\
\hline & Buli & 12.5 & $2.24(1.9-2.7)$ & $2.91(2.4-3.6)^{b}$ \\
\hline & Other & 7.5 & $1.27(1.0-16)$ & $0.98(0.8-1.3)$ \\
\hline \multirow[t]{2}{*}{ Location } & Rural & 6.2 & 1 & 1 \\
\hline & Urban & 7.9 & $1.28(1.2-1.4)$ & $1.29(1.1-1.5)^{b}$ \\
\hline \multirow[t]{3}{*}{ Household size } & 1 member & 10.7 & $1.83(1.5-2.2)$ & $1.36(1.1-1.8)^{\mathrm{b}}$ \\
\hline & 2-4 members & 7.2 & $1.19(1.1-1.3)$ & $1.36(1.1-1.8)^{\mathrm{b}}$ \\
\hline & $5+$ members & 6.1 & 1 & 1 \\
\hline \multirow[t]{5}{*}{ Socio-economic status } & Poorest & 6.3 & 1 & 1 \\
\hline & Poorer & 6.1 & $0.96(0.9-1.1)$ & $0.97(0.9-1.1)$ \\
\hline & Poor & 6.2 & $0.98(0.9-1.1)$ & $1.10(0.9-1.2)$ \\
\hline & Less poor & 6.3 & $1.00(0.9-1.1)$ & $1.04(0.9-1.2)$ \\
\hline & Least poor & 7.7 & $1.24(1.1-1.4)$ & $1.23(1.0-1.4)$ \\
\hline \multirow[t]{2}{*}{ Recent illness or injury } & Yes & 42.9 & $34.14(31.3-37.2)$ & $32.4(29.4-35.8)^{\mathrm{b}}$ \\
\hline & No & 3.8 & 1 & 1 \\
\hline \multirow[t]{3}{*}{ Self-assessed health status } & Poor or very poor & 25.8 & $5.49(6.5-8.9)$ & $2.08(1.7-2.5)^{b}$ \\
\hline & Good or very good & 5.9 & 1 & 1 \\
\hline & Don't know & 5.2 & $0.87(0.62-1.2)$ & $0.89(0.6-1.3)$ \\
\hline \multirow[t]{3}{*}{ Chronic medication } & Yes & 32.9 & $7.58(6.5-8.9)$ & $2.79(2.2-3.5)^{\mathrm{b}}$ \\
\hline & No & 93.9 & 1 & 1 \\
\hline & Don't Know & 7.4 & $1.2(0.6-2.4)$ & $0.82(0.3-1.9)$ \\
\hline
\end{tabular}

The reference group for the calculation of the odds ratio (OR) is indicated by one

NS not significant in both bivariate and multivariate analyses

${ }^{a}$ Representing infants who have not yet attained school going age in order to be in school

${ }^{\mathrm{b}}$ Significant in multivariate analyses 
Table 4 Factors associated with utilization of inpatient health services

\begin{tabular}{|c|c|c|c|c|}
\hline \multirow[t]{2}{*}{ Variable } & \multirow[b]{2}{*}{ Categories } & \multicolumn{3}{|c|}{ Inpatient health services } \\
\hline & & $\%$ Utilized & Unadjusted OR (95\% Cl) & Adjusted OR $(95 \% \mathrm{Cl})$ \\
\hline \multirow[t]{2}{*}{ Health insurance } & Insured & 1.4 & $3.49(2.8-4.4)$ & $2.78(2.2-3.6)^{b}$ \\
\hline & Uninsured & 0.4 & 1 & 1 \\
\hline \multirow[t]{6}{*}{ Age } & $0-4$ & 1.7 & $1.98(1.5-2.6)$ & $1.27(0.9-1.9)$ \\
\hline & $5-17$ & 0.5 & $0.57(0.4-0.7)$ & $0.66(0.5-0.9)$ \\
\hline & $18-34$ & 0.9 & 1 & 1 \\
\hline & $35-59$ & 1.4 & $1.63(1.3-2.0)$ & $1.53(1.2-1.9)^{\mathrm{b}}$ \\
\hline & $60-69$ & 1.4 & $1.65(1.2-2.3)$ & $1.41(0.9-2.1)$ \\
\hline & $70+$ (aged) & 1.6 & $1.88(1.3-2.7)$ & $1.65(1.1-2.5)^{b}$ \\
\hline \multirow[t]{2}{*}{ Gender } & Male & 0.9 & 1 & 1 \\
\hline & Female & 1.2 & $1.3(1.1-1.6)$ & $1.10(0.9-1.3)$ \\
\hline \multirow[t]{5}{*}{ Educational level } & No education & 1.1 & $0.61(0.4-0.9)$ & $1.03(0.7-1.6)$ \\
\hline & Primary & 0.5 & $0.29(0.2-0.4)$ & $0.84(0.5-1.3)$ \\
\hline & Secondary & 1.2 & $0.66(0.4-0.9)$ & $1.25(0.8-1.9)$ \\
\hline & Tertiary & 1.9 & 1 & 1 \\
\hline & Below school going age ${ }^{a}$ & 1.4 & $0.73(0.5-1.1)$ & $1.47(0.9-2.5)$ \\
\hline \multirow[t]{3}{*}{ Religion } & Traditional \& other & 0.7 & $0.49(0.4-0.7)$ & NS \\
\hline & Christianity & 1.3 & $0.87(0.6-1.2)$ & NS \\
\hline & Islam & 1.4 & 1 & NS \\
\hline \multirow[t]{4}{*}{ Ethnicity } & Kasem & 1.2 & 1 & 1 \\
\hline & Nankam & 0.8 & $0.66(0.6-0.8)$ & $0.95(0.8-1.2)$ \\
\hline & Buli & 1.6 & $1.41(0.9-2.2)$ & $1.71(1.1-2.7)^{\mathrm{b}}$ \\
\hline & Other & 2.4 & $2.12(1.4-3.1)$ & $1.17(0.8-1.8)$ \\
\hline \multirow[t]{2}{*}{ Location } & Rural & 0.9 & 1 & 1 \\
\hline & Urban & 2.2 & $2.62(2.2-3.1)$ & $1.32(1.0-1.7)$ \\
\hline \multirow[t]{3}{*}{ Household size } & 1 member & 2.4 & $2.7(1.9-3.9)$ & $1.55(1.0-1.5)$ \\
\hline & 2-4 members & 1.4 & $1.62(1.4-1.9)$ & $1.27(1.0-1.5)$ \\
\hline & $5+$ members & 0.9 & 1 & 1 \\
\hline \multirow[t]{5}{*}{ Socio-economic status } & Poorest & 0.7 & 1 & 1 \\
\hline & Poorer & 0.8 & $1.14(0.9-1.5)$ & $1.10(0.8-1.5)$ \\
\hline & Poor & 0.8 & $1.12(0.8-1.5)$ & $1.10(0.8-1.5)$ \\
\hline & Less poor & 1.2 & $1.73(1.3-2.3)$ & $1.48(1.1-1.9)^{\mathrm{b}}$ \\
\hline & Least poor & 2.3 & $3.40(2.6-4.4)$ & $2.10(1.5-2.9)^{b}$ \\
\hline \multirow[t]{2}{*}{ Recent illness or injury } & Yes & 0.8 & $8.23(6.9-9.9)$ & $5.72(4.6-7.1)^{\mathrm{b}}$ \\
\hline & No & 6.0 & 1 & 1 \\
\hline \multirow[t]{3}{*}{ Self-assessed health status } & Poor or very poor & 4.9 & $5.43(4.2-6.9)$ & $2.52(1.9-3.4)^{b}$ \\
\hline & Good or very good & 0.9 & 1 & 1 \\
\hline & Don't know & 0.7 & $0.77(0.32-1.9)$ & $0.81(0.3-2.0)$ \\
\hline \multirow[t]{3}{*}{ Chronic medication } & Yes & 8.8 & $10.27(7.9-13.4)$ & $3.48(2.5-4.8)^{\mathrm{b}}$ \\
\hline & No & 0.9 & 1 & 1 \\
\hline & Don't Know & 1.7 & $1.79(0.4-7.2)$ & $1.19(0.3-5.0)$ \\
\hline
\end{tabular}

The reference group for the calculation of the odds ratio (OR) is indicated by one

NS not significant in both bivariate and multivariate analyses

${ }^{\text {a }}$ Representing infants who have not yet attained school going age in order to be in school

${ }^{\mathrm{b}}$ Significant in multivariate analyses 
Table 5 Health insurance status and types of health care providers visited

\begin{tabular}{|c|c|c|c|c|c|c|c|}
\hline & \multirow[b]{2}{*}{ Provider } & \multicolumn{3}{|c|}{ Outpatient rate (per 1000) } & \multicolumn{3}{|c|}{ Inpatient rate (per 1000) } \\
\hline & & Insured & Uninsured & Total & Insured & Uninsured & Total \\
\hline \multirow[t]{6}{*}{ Population } & CHPS compounds (public) & 44.6 & 5.4 & 30.1 & 1.0 & 0.2 & 0.7 \\
\hline & Pharmacies or LCSs & 14.9 & 38.3 & 23.6 & 0.0 & 0.0 & 0.0 \\
\hline & Public health centres & 13.0 & 1.9 & 8.9 & 1.2 & 0.9 & 1.1 \\
\hline & Private clinics & 10.4 & 2.1 & 7.3 & 0.4 & 0.1 & 0.3 \\
\hline & Public hospitals & 19.3 & 1.8 & 12.8 & 23.4 & 3.0 & 15.9 \\
\hline & Traditional healers & 2.8 & 1.8 & 2.4 & 0.1 & 0.2 & 0.1 \\
\hline \multirow[t]{6}{*}{ Poorest } & CHPS compounds (public) & 56.3 & 6.9 & 33.3 & 0.5 & 0.0 & 0.3 \\
\hline & Pharmacies or LCSs & 12.2 & 35.6 & 23.4 & 0.0 & 0.0 & 0.0 \\
\hline & Public health centres & 20.2 & 2.8 & 12.1 & 2.0 & 1.8 & 1.9 \\
\hline & Private clinics & 6.1 & 1.5 & 4.0 & 0.3 & 2.2 & 1.2 \\
\hline & Public hospitals & 15.0 & 1.9 & 8.9 & 18.1 & 0.0 & 9.7 \\
\hline & Traditional healers & 2.7 & 2.4 & 2.6 & 0.1 & 0.0 & 0.1 \\
\hline \multirow[t]{6}{*}{ Least poor } & CHPS compounds (public) & 20.3 & 1.1 & 17.9 & 1.4 & 0.0 & 1.2 \\
\hline & Pharmacies or LCSs & 18.6 & 26.9 & 19.6 & 0.0 & 0.0 & 0.0 \\
\hline & Public health centres & 5.8 & 0.0 & 5.0 & 0.3 & 0.0 & 0.3 \\
\hline & Private clinics & 20.0 & 4.3 & 18.0 & 1.1 & 1.1 & 1.1 \\
\hline & Public hospitals & 35.0 & 3.2 & 31.0 & 38.3 & 3.2 & 33.8 \\
\hline & Traditional healers & 3.1 & 3.2 & 3.1 & 0.0 & 0.0 & 0.0 \\
\hline
\end{tabular}

utilization of health services under the communitybased health insurance [23-26]. Thus, the results from our study conducted in a rural poor setting do not differ from findings from other similar settings. A recent report from the Ministry of Health showed a continued rise in the utilization of health services under the NHIS. From the period 2003 to 2009 , outpatient attendance per capita rose from 0.50 to 0.81 [27]. The observed increased in the utilization of health services could be linked to the introduction of the NHIS. However, these findings could have financial implications for the sustainability of the NHIS, given that those more likely to need care are registered and subsequently utilizing health services, rather than all Ghanaians being registered and contributing financially.

The findings also suggest that the existence of the NHIS has led to increased use of the CHPS compounds by the poorest who were insured, as reported in other studies [28-30] Thus, distance as a barrier to the utilization of health services has been reduced with the introduction of the CHPS compounds. A recent report by the Ministry of Health indicated that CHPS compounds in Ghana were responsible for about $5 \%$ of the total outpatient attendance in 2011 [28]. We recommend that more CHPS compounds be built to help bring primary health services to the doorsteps of Ghanaians and therefore help strengthen the gatekeeper system and promote more broadly UHC.
Uninsured respondents, regardless of their socioeconomic standing, were utilizing outpatient health services from pharmacies or licensed chemical shops, requiring direct OOP payments with its attendant financial implications. We recommend a rigorous effort on the part of the NHIS to register more individuals in the NHIS in order to reduce OOP payments and to promote utilization. The findings show that socio-economic standing did not pose a barrier to outpatient health service use; rather, it influenced the type of health provider used. The study has helped to shed light on the type of health provider utilized by the insured and uninsured.

\section{Limitations of the study}

One of the limitations of this study was that household heads were responsible for answering all the questions for their household members. In the absence of the household head, an adult member of the household with information about the household members responded for the household members. It may be problematic for the household head or the representative to provide accurate responses for all household members, especially on their health status as well as their health-seeking behavior. However, considering that the household sizes are generally small (about five), and being a rural area where information is often shared among household members, we do not expect so much variation in the responses from individuals from the same household. 
As the study was cross-sectional, we are unable to determine changes in utilization across time. The sampled insured and uninsured households may systematically be different from each other due to unobserved factors such as some aspect of wealth or income, perceived benefits of insurance, and perceived health status which might influence registration with the NHIS and subsequent utilization of health services. Thus our findings may be biased by these unobservable factors. Nonetheless, the study showed a strong association between having an insurance cover and the utilization of health services. The implementation of the NHIS in Ghana provides important lessons for other low- and middleincome countries that are planning or have implemented prepayment programs in one way or the other.

\section{Conclusion}

Being insured with the NHIS is associated with increased utilization of both outpatient and inpatient health services compared to the uninsured. As expected, respondents considered to be in need of health services (those with poor or very poor self-reported health status, history of recent illness or injury, and those on chronic medication) were found to have increased odds of utilizing health services under the NHIS. The insured were using outpatient health services from CHPS compounds and that of inpatient health services from public hospitals. However, the uninsured utilized outpatient health services from pharmacies or licensed chemical shops. The NHIS can be an effective tool for achieving UHC and hence pragmatic efforts should be made to sustain it.

\section{Abbreviations \\ CHPS: Community-based Health Planning and Services; Cl: Confidence interval; LCSs: Licensed chemical shops; NHDSS: Navrongo Health and Demographic Surveillance System; NHIS: National Health Insurance Scheme; OOP: Out-of pocket; OR: Odds ratio; UHC: Universal health coverage; WHO: World Health Organization}

\section{Acknowledgements}

We thank the respondents who offered their time and energies to be part of the study. We are also grateful to INDEPTH Network Secretariat for coordinating the project.

\section{Funding}

The Rockefeller Foundation provided the funds for the study (Health Systems Project grant number 2011THS341), but had no role in the writing and publication of this manuscript.

\section{Availability of data and materials}

The datasets generated during and/or analysed during the current study are not publicly available. The datasets are privately owned by the Navrongo Health Research Centre, Navrongo, Ghana, but are available from the corresponding author on reasonable request.

\section{Authors' contributions}

JA, PW, OS, JG, and PAD conceived and designed the study. JA, PW, and PAD carried out the study and analysed, interpreted, and wrote the paper. JA, AK, MB, AO, OS, and JG critically reviewed the manuscript. All authors read and approved the final manuscript.

\section{Ethics approval and consent to participate}

The Institutional Review Board (IRB) of the Navrongo Health Research Centre, Ghana, reviewed and approved the study. Heads of households or their representatives gave verbal approval for the interviews to be conducted.

\section{Consent for publication}

Heads of households or their representatives gave verbal consent at the time of the interviews for the publication of the findings.

\section{Competing interests}

The authors declare that they have no competing interests.

\section{Publisher's Note}

Springer Nature remains neutral with regard to jurisdictional claims in published maps and institutional affiliations.

\section{Author details}

${ }^{1}$ Navrongo Health Research Centre, Ghana Health Service, Navrongo, Ghana. ${ }^{2}$ Kintampo Health Research Centre, Ghana Health Service, Kintampo, Ghana. ${ }^{3}$ INDEPTH Network Secretariat, Accra, Ghana. ${ }^{4}$ Centre for Health Policy, School of Public Health, Faculty of Health Sciences, University of the Witwatersrand, Johannesburg, South Africa.

Received: 12 October 2016 Accepted: 27 November 2017

/ Published online: 13 December 2017

References

1. WHO. Tracking UHC: first global monitoring report. Geneva: World Health Organization and World Bank; 2015.

2. WHO. Constitution of the World Health Organization. Am J Public Health Nations Health. 1946;36(11):1315-23. PubMed PMID: PMC1625885

3. NHIA. National Health Insurance Authority 2012 annual report. Accra: National Health Insurance Authority; 2012.

4. NHIA. NHIS subscriber handbook: a guide to registering and accessing NHIS Accra: National Health Insurance Authority; 2015.

5. NHIA. Ghana health insurance review: The National Health Insurance Scheme magazine. Accra: National Health Insurance Authority; 2012.

6. Morestin F, Ridde V. How can the poor be better integrated into health insurance programs in Africa? An overview of possible strategies. Canada: Universite de Montreal; 2009.

7. Jehu-Appiah C, Aryeetey G, Spaan E, de Hoop T, Agyepong I, Baltussen R. Equity aspects of the National Health Insurance Scheme in Ghana: who is enrolling, who is not and why? Soc Sci Med. 2011;72(2):157-65. doi:10.1016/ j.socscimed.2010.10.025. Epub 18 Nov 2010.

8. Akazili J, Garshong B, Aikins M, Gyapong J, Mclntyre D. Progressivity of health care financing and incidence of service benefits in Ghana. Health Policy Plan. 2012;27(suppl 1):i13-22. doi:10.1093/heapol/czs004.

9. Akazili J, Welaga P, Bawah A, Achana F, Oduro A, Awoonor-Williams J, et al. Is Ghana's pro-poor health insurance scheme really for the poor? Evidence from northern Ghana. BMC Health Serv Res. 2014;14(1):1-9. doi:10.1186/ s12913-014-0637-7.

10. Dixon J, Tenkorang E, Luginaah I. Ghana's National Health Insurance Scheme: helping the poor or leaving them behind? Environ Plann C: Gov Policy. 2011;29:1102-15.

11. Kanchebe Derbile E, van der Geest S. Repackaging exemptions under National Health Insurance in Ghana: how can access to care for the poor be improved? Health Policy Plan. 2012;28:586-95.

12. GOG. The Livelihood Empowerment Against Poverty (LEAP) Programme: reducing poverty and promoting growth in Ghana. Accra: Ministry of Gender, Children and Social Protection; 2013.

13. Dixon J, Luginaah I, Mkandawire P. The National Health Insurance Scheme in Ghana's UpperWest region: a gendered perspective of insurance acquisition in a resource-poor setting. Soc Sci Med. 2014;122:103-12.

14. Dalaba MA, Akweongo P, Aborigo RA, Ataguba JE. To insure or not to insure: the influence of insurance status on health seeking behaviour in the KassenaNankana district of Ghana. African Journal of Health Sciences. 2012; 21:161-73.

15. Al-Hassan R, Diao X. Regional disparities in Ghana: policy options and public investment implications. Washington: International Food Policy Research Institute, Sustainable Solutions for Ending Hunger and Poverty IFPRI; 2007. Discussion paper February 2007 
16. UNDP. Kassena-Nakana District human development report 2010: resource endowment, investment opportunities and the attainment of MDGs. Accra: United Nations and Development Programme; 2010.

17. Debpuur C, Welaga P, Wak G, Hodgson A. Self-reported health and functional limitations among older people in the Kassena-Nankana District, Ghana. Glob Health Action. 2010;5(10)

18. StataCorp. Stata statistical software: release 12. College Station: StataCorp LP; 2011.

19. Habib SS, Perveen S, Khuwaja HMA. The role of micro health insurance in providing financial risk protection in developing countries-a systematic review. BMC Public Health. 2016;16(1):1-24. doi:10.1186/s12889-016-2937-9.

20. Dalinjong $P$, Laar $A$. The national health insurance scheme: perceptions and experiences of health care providers and clients in two districts of Ghana. Heal Econ Rev. 2012;2(1):13. doi:10.1186/2191-1991-2-13.

21. Blanchet NJ, Fink G, Osei-Akoto I. The effect of Ghana's National Health Insurance Scheme on health care utilisation. Ghana Med J. 2012;46(2):76-84. PubMed PMID: PMC3426378

22. Sekyi S, Domanban PB. The effects of health insurance on outpatient utilization and health care expenditure in Ghana. Int J Humanit Soc Sci. 2012;2(10 (Special Issue-May 2012)):40-9.

23. Mejía-Guevara I, Hill K, Subramanian SV, Lu C. Service availability and association between Mutuelles and medical care usage for under-five children in rural Rwanda: a statistical analysis with repeated cross-sectional data. BMJ Open. 2015;5(9):e008814.

24. Lu C, Chin B, Lewandowski JL, Basinga P, Hirschhorn LR, Hill K, et al. Towards universal health coverage: an evaluation of Rwanda Mutuelles in its first eight years. PLoS One. 2012;7(6):e39282. doi:10.1371/journal.pone. 0039282. PubMed PMID: PMC3377670

25. Ruhara CM. Effect of health insurance on demand for outpatient medical care in Rwanda: an application of the control function approach. Rwanda J. 2016;1

26. Saksena P, Antunes AF, Xu K, Musango L, Carrin G. Mutual health insurance in Rwanda: evidence on access to care and financial risk protection. Health Policy. 2011;99(3):203-9. doi:10.1016/j.healthpol.2010.09.009. PubMed PMID: 20965602. Epub 23 Oct 2010

27. MOH. The health sector in Ghana: facts and figures 2010. Accra: Ministry of Health, Ghana; 2010

28. MOH. National Community Health Planning and Services (CHPS) Policy: accelerating attainment of universal health coverage and bridging the access inequity gap. Accra: Ministry of Health, Ghana; 2014

29. Sakeah E, Doctor HV, McCloskey L, Bernstein J, Yeboah-Antwi K, Mills S. Using the community-based health planning and services program to promote skilled delivery in rural Ghana: socio-demographic factors that influence women utilization of skilledd attendants in Northern Ghana. BMC Public Health. 2014;14:344.

30. Nyonator FK, Awoonor-Williams KJ, Phllips JF, Tanya JC, Miller RA. The Ghana community-based health planning and services initiative for scaling up service delivery innovation. Health Policy Plan. 2005;20(1):25-34.

\section{Submit your next manuscript to BioMed Central and we will help you at every step:}

- We accept pre-submission inquiries

- Our selector tool helps you to find the most relevant journal

- We provide round the clock customer support

- Convenient online submission

- Thorough peer review

- Inclusion in PubMed and all major indexing services

- Maximum visibility for your research

Submit your manuscript at www.biomedcentral.com/submit

CBiomed Central 